\title{
Integrating Trust and Competency Management to Improve Learning
}

\author{
Nicola Capuano ${ }^{\dagger}$, Matteo Gaeta ${ }^{\dagger}$, Giuseppina Rita Mangione*, Francesco Orciuoli ${ }^{\dagger}$, Pierluigi Ritrovato* \\ *MOMA S.p.A. \\ 84081 Baronissi (SA), Italy \\ ${ }^{\dagger}$ Department of Electronic Engineering and Information Engineering \\ University of Salerno, 84084 Fisciano (SA), Italy \\ Email: \{gaeta, orciuoli\}@diima.unisa.it, \{ncapuano\}@unisa.it, \{mangione, ritrovato\}@momanet.it
}

\begin{abstract}
Nowadays, the importance of knowledge management is well understood by managers in the organizations and, at the same time, the great significance of trust, in enabling effective knowledge sharing, is emerging. Presence or lack of trust can have serious implications for organizations with respect to the quality and of their business processes. Several scientific works have confirmed the direct correlation between social-cognitive capital, in terms of competencies and experiences, and a feeling of trust in both learning and working collaborative environments. On the other hand, Competency-Based Management allows organizations to link human resources processes to competencies in order to shape its workforce capabilities and to achieve better results. Typically, employees' competencies (and proficiency levels) are stored and used by specific enterprise software, whereas the trust is not considered or left to managers' feeling. This work proposes an approach to the improvement of collaborative learning activities by refining and allowing (at workers' level) competency-finding processes through the social calculus of trust-in-competencies degree.
\end{abstract}

Keywords-corporate learning; trust; competency-based management; semantic web; social networking;

\section{INTRODUCTION}

\section{A. Background and Motivations}

According to [1], it is possible to define the trust as a mental state comprising expectacy (the trustor expect a specific behavior from the trustee) and belief. Trust is considered one of the main assets available for organizations to proactively manage the knowledge sharing and increase the knowledge capital. In literature, many contributions suggesting a correlation between social-cognitive capital and feel of trust in collaborative environment [2], with regard to working and learning activities [3], have been recognized. According to Ardichvili et al. [4] the lack of trust in competencies may be identified as one of the main barriers to knowledge sharing in online communities of practice discouraging any form of motivation and participation in work activities. Moreover, one's degree of trust in the competence of a community is positively related to one's engagement in knowledge sharing with the community. Recent studies have confirmed this hypothesis [5] and argue that social motivation in the workplace increases the growth of trustworthiness and directly impacts on employees' performances. Although several works providing models for trust management in virtual communities and social networks are reviewed in literature [6], they are not related to the trust in competencies the workers need to perform specific jobs. Competencies are indentified, classified and managed by a set of processes, namely Competency-Based Management (CBM), ensuring that there is the right person in the right position at the right time in order to achieve better results. Typically, CBM processes are top-down organizational processes supported by enterprise software (e.g. Human Resource Information Systems, etc.) that cannot be exploited to support team building operations in non-formal collaborative learning (and working) activities. The main goal of this work is to define an approach to enrich CBM with trust information in order to support (by enabling competency finding operations) and to improve (by considering trust as a way to increase the quality of knowledge sharing) collaborative learning (and working) activities at the workplace. In order to achieve the aforementioned goal, a semantic-annotated trust-based corporate social network (where nodes are the employees' profiles and arcs are the relationships among the employees) will be defined. The social nature of the network allows to overcome the limitation of traditional CBM by enabling bottom-up competency management operations improved with trust information. Furthermore, trust in competencies will be calculated by using a social approach, i.e. considering direct interactions between two workers and using semanticannotated social network to propagate trust degrees.

\section{B. A Brief Overview of the Proposed Approach}

This work proposes a trust-based corporate social network model (Section II) to improve learning (and working) processes regarding human resources in organizations. The idea underlying the proposed approach consists in defining a methodology to calculate the degree of trust a worker has in his/her colleagues' specific competencies. Let us assume the trustor's name is $a$ and the trustee's name is $c$. The calculus of trust degree is directly (by analysing the interactions between $a$ and $c$ ) performed if there has been at least one direct experience in which $a$ had evidence of a specific competency of $c$, otherwise the trust degree is performed by propagation using the network. In the case of 
propagation, for instance, let us also assume the existence of a worker $b$. $b$ trusts $c$ in a specific competency, $a$ trusts $b$ in his/her belief about the aforementioned specific competency, so it is possible to calculate the trust of $a$ in the specific competency of $c$. Ontologies, taxonomies and vocabularies (modelled with Semantic Web schemes and languages) are used to define a trust-based corporate social network (section II) where employees' profiles and competencies, workplace relationships and information about trust in colleagues' competencies and believes are explicitly asserted. The network will be queried in order to support decision-making tasks (at both organizational and worker's level) regarding learning and working activities. The proposed approach includes the contextualisation (realized by taking care of work activities modelled in section III) of an existing method to perform the calculus of trust degree both directly (section IV) and by propagation (section V). Lastly, a brief discussion about the advantages of the proposed approach is provided (section VI).

\section{TRust-BASEd Corporate Social Network}

In this section, a model of employees' profiles network is described by integrating and extending existing Semantic Web schemes, based on the W3C Semantic Web stack ${ }^{1}$. The main result reported in this section is the definition of the Trust in Competencies and Trust in Belief model and its correlation with the employees' profiles in order to build the Trust-based Corporate Social Network.

\section{A. Competency Model}

According to the Professional Learning Ontology and Competencies (PLOC) described in [7], competencies are defined as abstractions for making human Knowledge, Skills and Abilities (KSA) manageable and addressable. Before competencies can be managed (by means of CBM processes), they should be classified and their context defined. It seems to be a good choice to divide them into behavioural and technical competencies (see fig. 2). Behavioural competencies include all those soft skills and attitudes that enable a person to perform well in a specific function, such as communication, problem-solving, integrity, planning and organisation, etc. Technical competencies are specific and depending on the job. These latter could range, for instance, from the knowledge of software development methodologies to skill for Java programming. Typically, competencies are organized in a hierarchy of four levels. Main classes and properties ragarding the organization of competencies in PLOC are shown in fig. 1.

\section{B. Employees' Profiles and Relationships}

In order to model the employees' profiles, at least personal and curriculum vitae data have to be considered. In

\footnotetext{
${ }^{1}$ http://www.w3.org/standards/semanticweb/
}

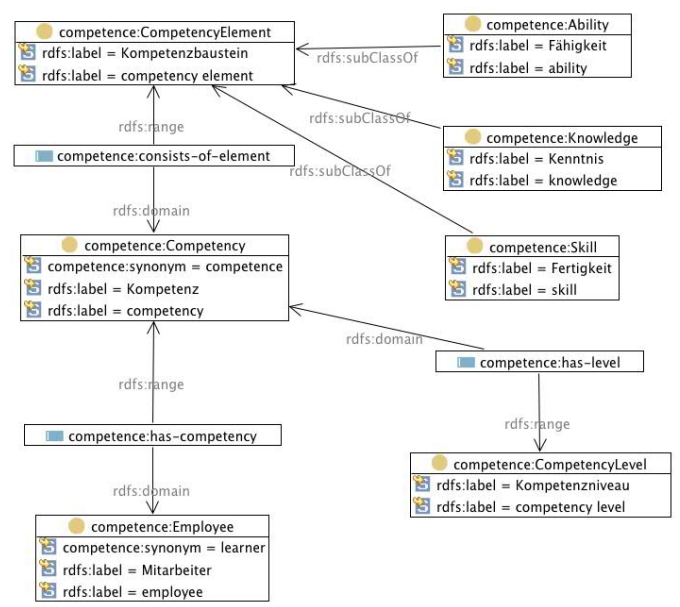

Figure 1. Organization of competencies in PLOC.

[8] an integrated model constitued by $\mathrm{FOAF}^{2}$ (organizing basic employees' information), ResumeRDF $^{3}$ (modeling the curriculum vitae of employees) and PLOC (modeling competencues and their link with the employees) is presented. In particular, the foaf:Person class is crucial to model part of personal information about an employee. Some useful properties are foaf:firstName, foaf:surname, foaf:mbox, foaf:publications, foaf:interest, etc. Moreover, ResumeRDF is a RDF metadata vocabulary mainly used to describe work and education history of the employees. The main part of the ResumeRDF model is the $c v: C V$ class that mantains information about a specific employee ( $c v$ :Person), his (her) education (cv:Education), his (her) work history ( $c v$ :WorkHistory), and so on. Although, ResumeRDF provides a way to model skills ( $c v$ :Skill) owned by an employee, it is preferable to use another scheme in order to adhere to a more complete definition (KSA) of competency (see paragraph II-A) provided by PLOC. The PLOC defines competence:Employee class and competence:has-competency property that are useful to link an employee with its competencies. In order to link foaf:Person with $c v$ :Person and competence:Employee, the subclassing operation is used. In particular, competence:Employee becomes a subclass of $c v$ :Person that becomes a subclass of foaf:Person. The effect of the application of the subclassing operation is that all individuals of type competence:Employee are also instances of $c v$ :Person class and instances of foaf:Person class. So, all properties of foaf:Person are inherited by $c v$ :Person and all properties of these two classes are inherited by competence:Employee class. One of the most important FOAF property is foaf:knows that links two instances of the foaf:Person class by building a semantic-annotated social network where the employees are nodes and their rela-

\footnotetext{
${ }^{2}$ http://www.foaf-project.org/

${ }^{3}$ http://rdfs.org/resume-rdf/
} 
tionships are arcs. However, in the corporate context, we need richer semantics to model relationships among employees. The RELATIONSHIP ${ }^{4}$ vocabulary represents one of the plausible solution for the aforementioned problem. In particular, relationship:worksWith (asserts that person $e$ works for the same employeer of person $d$ ) and relationship:collaboratesWith (asserts that person $e$ has worked at least one time on the same activity with person $d$ ) are important for our purpose. The two aforementioned properties can be applied between two foaf:Person instances and they are symmetrical.

\section{Trust in Competencies and Trust in Belief}

The employees' profiles network, described with Semantic Web schemes, has to be enriched with information regarding trust. In particular, in [9] the authors build a formalsemantics-based model for the calculus of trust and refer to two basic types of trust: trust in performance and trust in belief. The first one is the trust about what the trustee performs, whereas the second one is the trust about what the trustee believes. The present work starts from the results of [9] and extends them in order to deal with the concept of trust in competencies. In particular, we propose the following statements:

$$
\begin{aligned}
& \operatorname{trust}_{c}(d, e, \operatorname{apply}(e, x), k), \\
& \operatorname{trust}_{b}(g, d, \operatorname{apply}(e, x), k) .
\end{aligned}
$$

The statement 1 indicates that employee $d$ (trustor) trusts employee $e$ (trustee) regarding the capability of $e$ to apply competency $x$ in context $k$. The statement 2 indicates that employee $g$ (trustor) trusts employee $d$ (trustee) regarding $d$ 's belief about the capability of $e$ to apply competency $x$. Note that, in the Semantic Web-based modeling, $d, e$ and $g$ are individuals of type competence:Employee, $x$ is an individual of typecompetence:Competency, whereas $k$ can be interpret as an instance of a skos:Concept. SKOS ${ }^{5}$ is a Semantic Web scheme used to build taxonomies and controlled vocabularies. For the aim of this work, SKOS will be used to model a controlled vocabulary of contexts of interest in a given organization using skos:narrower and skos:broader properties to relate instances of skos:Concept.

The table in Fig. 2 shows variants of 1 and 2 for all competency types.

Now, a Semantic Web-based scheme is needed in order to model trust statements of Fig. 2. The Trust Ontology ${ }^{6}$. The Trust Ontology is a small ontology, written on OWL, that allows people to say how much they trust in other people. It is defined as an extension of the FOAF scheme. The most important class of the Trust Ontology is trust:TopicalTrust. The

\footnotetext{
${ }^{4}$ http://vocab.org/relationship/.html

${ }^{5}$ www.w3.org/2004/02/skos/

\begin{tabular}{|c|c|c|c|}
\hline Category & Sub-category & Examples & Statement \\
\hline \multirow{6}{*}{\begin{tabular}{|l} 
Technical \\
Competencies
\end{tabular}} & \multirow[t]{3}{*}{ Knowledge } & Java language syntax & \multirow{3}{*}{$\begin{array}{l}\text { trust_c(d, e, use }(e, x), k) \text {, } \\
\text { trust_b(g, d, use }(e, x), k)\end{array}$} \\
\hline & & \begin{tabular}{|l} 
Description logics \\
\end{tabular} & \\
\hline & & UML notation & \\
\hline & \multirow[t]{3}{*}{ Technical Skills } & Java programming & \multirow{3}{*}{$\begin{array}{l}\text { trust_c(d, e, apply(e, } x), k) \\
\text { trust_b(g, d, apply(e, } x), k)\end{array}$} \\
\hline & & OWL modeling & \\
\hline & & Project management & \\
\hline \multirow{14}{*}{$\begin{array}{l}\text { Behavioural } \\
\text { and Cognitive } \\
\text { Competencies }\end{array}$} & \multirow[t]{3}{*}{ Thinking Skills } & \begin{tabular}{|l|} 
Problem-solving \\
\end{tabular} & \multirow{3}{*}{$\begin{array}{l}\text { trust_c(d, e, apply(e, x), k), } \\
\text { trust_b(g, d, apply(e, x), k) }\end{array}$} \\
\hline & & Innovation \& creativity & \\
\hline & & Continuous learning & \\
\hline & \multirow[t]{4}{*}{\begin{tabular}{|l} 
Interpersonal Skills \\
\end{tabular}} & Teamwork & \multirow{4}{*}{$\begin{array}{l}\text { trust_c(d, e, apply(e, x), k), } \\
\text { trust_b(g, d, apply(e, x), k) }\end{array}$} \\
\hline & & Relationship management & \\
\hline & & Communication & \\
\hline & & Teaching others & \\
\hline & \multirow[t]{3}{*}{ Action Skills } & Decision-making & \multirow{3}{*}{$\begin{array}{l}\text { trust_c(d, e, apply(e, } x), k), \\
\text { trust_b(g, d, apply }(e, x), k)\end{array}$} \\
\hline & & Results orientations & \\
\hline & & Planning \& organization & \\
\hline & \multirow[t]{4}{*}{ Personal Qualities } & Integrity & \multirow{4}{*}{$\begin{array}{l}\text { trust_c(d, e, reveal( }(\mathrm{e}, \mathrm{x}), \mathrm{k}) \text {, } \\
\text { trust_b(g, d, reveal }(\mathrm{e}, \mathrm{x}), \mathrm{k})\end{array}$} \\
\hline & & Self-motivation & \\
\hline & & Responsibility & \\
\hline & & Self-management & \\
\hline
\end{tabular}

${ }^{6} \mathrm{http} / /$ trust.mindswap.org/trustOnt.shtml
}

Figure 2. Managing different types of competencies

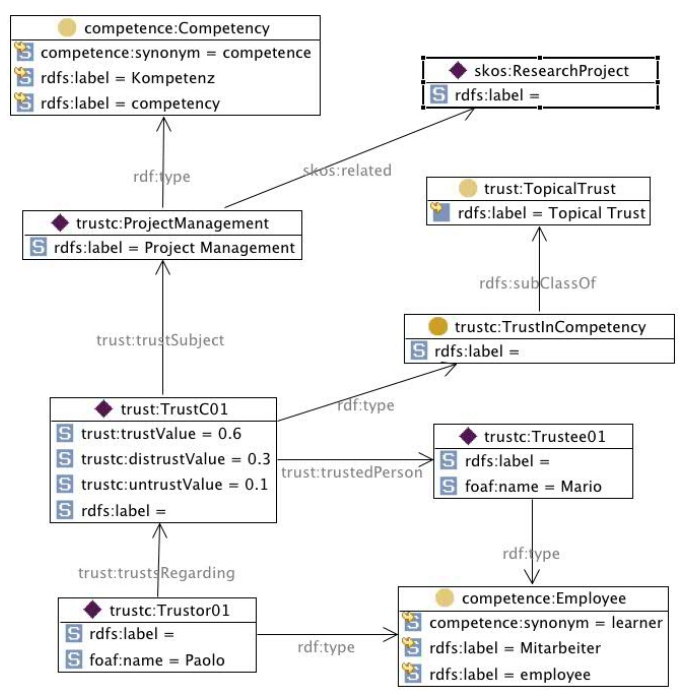

Figure 3. Extended Trust Ontology.

classes trustc:TrustInCompetency and TrustInBelief have been defined by subclassing trust:TopicalTrust in order to respectively model trust in competencies and trust in belief. Subclasses of trustc:TrustInCompetency could be defined in order to model all types of competencies illustrated in Fig. 2. The individuals of trustc:TrustInCompetency allow to link an instance of competence:Employee (trustor) with another instance of competence:Employee (trustee) and with the specific target of the trust, i.e. an individual of type competence:Competency. The data property trust:trustValue is used to assign a value to the trust in the target competency of the trustee. Fig. 3 shows how the extended Trust Ontology is instantiated.

It is important to note that in origin the class trust:TopicalTrust did not support values for distrust (that is the opposite of trust) and untrust (that is the status in which the degree of confidence is insufficient to trust) in order to solve some reasoning problems. We have extended the Trust 
Ontology with the new data properties trustc:distrustValue and trustc:untrustValue. In the extended ontology, trust, distrust and untrust have values in $[0,1]$. The graph in fig. 3 states that Paolo trusts Mario, with value 0.6 , to apply the Project Management competency (for sake of simplicity the graph does not report the competency level) in the context ResearchProject. The modelling of a statement for trust in belief is similar to that for trust in competencies. In this case of trust in belief, the property trust:trustSubject does not link a competency, it links a context (an individual of skos:Concept).

\section{WORK ACTIVITIES}

In order to calculate trust, distrust and untrust degrees it is important to understand how organizational activities are modelled. In particular, the PLOC provides the competence:OrganizationalEntity class. The competence:Task subclass of competence:OrganizationalEntity can be used as a basis to define a work activity in which an employee (worker) executes a specific task by means of the competence:is-in property (with domain competence:OrganizationalEntity and range competence:Employee). Furthermore, the competence:requires-competency property (with domain competence:OrganizationalEntity and range competence:Competency) is needed to indicate the competencies that are required in order to successfully carry out a specific task. In order to complete the Work Activities model we need to include a supervisor and the possibility for the supervisor to review the performance of the worker. So, the trustc:supervises property and the $R D F$ Review Vocabulary ${ }^{7}$ have to be introduced. The first one is used in order to relate instances of competence:Task class (supervised tasks) with instances of competence:Employee (supervisors). The second one provides a set of classes and properties useful to attach a review (rev:Review class) to a resource (in this case the resource will be an individual of type competence:Task). The underlying idea is that the supervisor assigns a rate (rev:rating is a numeric property with domain rev:Review) to the performance of the employee involved in the task. The rate can be interpreted as a value indicating the how the required competencies have been applied during the execution of the task. Supervisor and worker are both individuals of competence:Employee class.

\section{Calculus of Trust, Distrust and Untrust}

In this section, a method to calculate degrees for trust, distrust and untrust is proposed. For this aim, the probabilistic approach illustrated by the authors in [9] has been adapted for context and purpose of this work. The aforementioned contextualisation and the use of semantic-annotated data are

\footnotetext{
${ }^{7}$ http://danja.talis.com/xmlns/rev_20071109/index.html
}

advancements with respect to the results of [9]. Trust is interpreted as a probability distribution over three mental states (believing, disbelieving and undecidable) corresponding to trust, disrtust and untrust. Intuitively, the trust $\alpha$ of $e$ (trustor) for $d$ (trustee) in successfully applying a competency $x$ in context $k$ can be approximated with the probability that $e$ will successfully apply $x$ in context $k$. Distrust $\beta$ is the opposite of trust. Instead, unstrust $\gamma$, corresponding to the third state of the probability distribution, can be calculated by using the formula $\alpha+\beta+\gamma=1$. Authors in [9] propose to calculate probability measures in terms of frequencies of positive experiences between the trustor and the trustee. More in details, in the case of trust in competencies the following formula (3) can be used.

$$
\alpha=t d_{c}(d, e, \operatorname{apply}(e, x), k)=n / m
$$

where $m$ is the total number of situations, experienced by $d$ (trustor), in which $e$ (trustee) has applied competency $x$ in the context $k$, and $n$ is the number of the trustor's positive experiences in that set, $\beta$ (distrust) can be calculated with the same formula (3) of $\alpha$ by replacing $n$ with $l$ and $t d_{c}$ with $d t d_{c}$, where $l$ is the number of negative experienes in the same set. Values for $n$ and $l$ can be calculated with the following:

$$
n=\sum_{m}^{i=1} e_{p}(i), l=\sum_{m}^{i=1} e_{n}(i),
$$

where $e_{p}(i), e_{n}(i) \in[0,1]$, representing the degree of the experience $i$ being positive or negative separately, and $e_{p}(i)+e_{n}(i) \leq 1$. In the context of this work, a positive (negative) experience of a trustor with a trustee is represented by a task, that requires competency $x$, where the supervisor (trustor) $d$ has provided a positive (negative) review rate $e_{p}(i)\left(e_{n}(i)\right)$ to the worker (trustee) $e$ for the application of competency $x$ in the context $k$. Moreover, in the case of trust in belief the following equations can be used.

$$
\alpha=d t_{b}(g, d, k)=n^{\prime} / m^{\prime},
$$

where $d t_{b}(g, d, k)$ approximates $d t_{b}(g, d, \operatorname{apply}(e, x), k), m^{\prime}$ is the total number of experiences observed by $g$ (trustor), in which $d$ (trustee) has applied any competency in context $k$, and $n^{\prime}$ is the number of positive experiences in that set. $\beta$ (distrust) can be calculated by using the same formula (5) and replacing $n^{\prime}$ with $l^{\prime}$, where $l^{\prime}$ is the number of negative experiences in the same set.

$$
n^{\prime}=\sum_{m^{\prime}}^{i=1} e_{p}^{\prime}(i), l^{\prime}=\sum_{m^{\prime}}^{i=1} e_{n}^{\prime}(i)
$$


where $e_{p}^{\prime}(i), e_{n}^{\prime}(i) \in[0,1]$, representing the degree of the experience $i$ being positive or negative separately, and $e_{p}^{\prime}(i)+e_{n}^{\prime}(i) \leq 1$. In this case, a positive (negative) experience of a trustor with a trustee is represented by a task where the supervisor (trustor) $g$ has provided a positive (negative) review rate $e_{p}^{\prime}(i)\left(e_{n}^{\prime}(i)\right)$ to the worker (trustee) $d$ for the application of any competency in the context $k$.

\section{Propagation of Trust}

In the previous section (IV) a method to calculate trust, distrust and untrust, between two nodes (employees) in the network, is provided. Now, a model of propagation of trust degrees in social networks [10] has to be exploited in order to calculate the trust in competencies between employee $e$ and employee $d$ also if $e$ has never supervised a task performed by $d$ in which $d$ has used the required competency $x$ in context $k$. In this case, it is possible to use the corporate social network and trust in belief to propagate trust in competencies (and also trust in belief) by contextualising the approach presented in [9]. In fig. ??, a piece of the semanticannotated corporate social network is depicted. In particular, for employee $e$ there are not values for trust, distrust and untrust for competency $x$ of employee $g$ in context $k$. But, it is possible to obtain the aforementioned values by exploiting trust in belief (in context $k$ ) of $e$ for $d$ and trust in competency $x$ of $d$ for $g$ in context $k$. In ?? only degrees for trust and distrust are indicated. In particular $t d_{c}$ and $d t d_{c}$ are values for $t d_{c}(d, g, \operatorname{apply}(x, k))$ and $d t d_{c}(d, g, \operatorname{apply}(x, k))$, $t d_{b}$ and $d t d_{b}$ are values for $t d_{b}(e, g, k)$ and $d t d_{b}(e, g, k)$. $p t d_{c}$ and $p d t d_{c}$ are values calculated by using the formulas 7 and 8.

$$
\begin{array}{r}
p t d_{c}=t d_{b}(e, d, k) \cdot t d_{c}(d, g, \operatorname{apply}(x, k)) \\
+d t d_{b}(e, d, k) \cdot d t d_{c}(d, g, \operatorname{apply}(x, k)), \\
p d t d_{c}=d t d_{b}(e, d, k) \cdot t d_{c}(d, g, \operatorname{apply}(x, k)) \\
+t d_{b}(e, d, k) \cdot d t d_{c}(d, g, \operatorname{apply}(x, k)) .
\end{array}
$$

The formulas 7 and 8 can be used to propagate trust, distrust and unstrust by considering paths of any length over the corporate social network. Trust in belief is propagated by replacing, in $7, t d_{c}(d, g, \operatorname{apply}(x, k))$ and $d t d_{c}(d, g, \operatorname{apply}(x, k))$ with $t d_{b}(d, g, k)$ and $d t d_{b}(d, g, k)$. The propagation of distrust degrees is obtained in the same way by modifying formula 8 .

\section{Final Remarks}

The proposed approach enables several scenarios in which competency-finding is executed by workers that, when introduced in project teams, can exploit feelings of trust in their peers' capabilities in order to activate help seeking processes. The competency search operation is refined by calculating trust degrees. For instance, a non-formal learning activity, like peer-coaching, is improved (trust improves learning as asserted in Section I) when the coach is selected by the worker by using, not only the required competencies as search parameters, but also the trust degree of the learner for the potential coach. The approach will be experimented and exploited in ARISTOTELE project to develop a trustbased Decision Support System for team building.

\section{ACKNOWLEDGMENT}

This research is partially supported by the European Commission under the Collaborative Project ARISTOTELE "Personalised Learning \& Collaborative Working Environments Fostering Social Creativity and Innovations Inside the Organisations", VII Framework Program, Theme ICT2009.4.2 (Technology-Enhanced Learning), Grant Agreement n. 257886

\section{REFERENCES}

[1] R. C. Mayer, J. H. Davis, and F. D. Schoorman, "An Integrative Model of Organizational Trust," The Academy of Management Review, vol. 20, no. 3, pp. 709-734, 1995. [Online]. Available: http://dx.doi.org/10.2307/258792

[2] N. Coppola, S. Hiltz, and N. Rotter, "Building trust in virtual teams," Professional Communication, IEEE Transactions on, vol. 47, no. 2, pp. 95 - 104, 2004.

[3] A. Abdul-Rahman and S. Hailes, "Supporting trust in virtual communities," in System Sciences, 2000. Proceedings of the 33rd Annual Hawaii International Conference on, 2000, p. 9 pp. vol.1.

[4] A. Ardichvili, V. Page, and T. Wentling, "motivation and barriers to participation in virtual knowledge-sharing communities of practice," Knowledge Management, vol. 7, no. 1, pp. 64-77, 2003.

[5] A. Grant and J. Sumanth, "Mission possible? the performance of prosocially motivated employees depends on manager trustworthiness." J Appl Psychol, vol. 94, no. 4, pp. 927-44, 2009.

[6] D. Artz and Y. Gil, "A survey of trust in computer science and the semantic web," J. Web Sem., vol. 5, no. 2, pp. 58-71, 2007.

[7] A. Schmidt and C. Kunzmann, "Towards a Human Resource Development Ontology for Combining Competence Management and Technology-Enhanced Workplace Learning," in 1st Workshop on Ontology Content and Evaluation in Enterprise (OntoContent 2006), vol. 4278. Springer, 2006, pp. 10781087.

[8] G. Acampora, M. Gaeta, F. Orciuoli, and P. Ritrovato, "Exploiting semantic and social technologies for competency management," Advanced Learning Technologies, IEEE International Conference on, vol. 0, pp. 297-301, 2010.

[9] J. Huang and D. Nicol, "A formal-semantics-based calculus of trust," Internet Computing, IEEE, vol. 14, no. 5, pp. 38 $-46,2010$.

[10] C.-N. Ziegler and G. Lausen, "Propagation models for trust and distrust in social networks," Information Systems Frontiers, vol. 7, no. 4-5, pp. 337-358, 2005. 